\title{
MicroRNA-135b has a neuroprotective role via targeting of $\beta$-site APP-cleaving enzyme 1
}

\author{
YI ZHANG ${ }^{1}$, HONGXIA XING ${ }^{1}$, SHUANGXI GUO ${ }^{1}$, ZHIYONG ZHENG $^{1}$, HAOLIANG WANG $^{1}$ and DAWEI XU ${ }^{2}$ \\ Departments of ${ }^{1}$ Neurology and ${ }^{2}$ Neurosurgery, The First Affiliated Hospital of Xinxiang Medical University, \\ Weihui, Henan 453100, P.R. China
}

Received June 3, 2015; Accepted January 15, 2016

DOI: $10.3892 / \mathrm{etm} .2016 .3366$

\begin{abstract}
MicroRNAs (miRs) are a class of endogenous small non-coding RNAs that have been revealed to negatively mediate the expression of their target genes at the post-transcriptional level. Recently, particular miRs have demonstrated an involvement in the pathogenesis of Alzheimer's disease (AD). However, the specific role of miR-135b in AD has yet to be elucidated. The present study aimed to investigate the neuroprotective role of miR-135b, in addition to its underlying mechanism. Herein, reverse transcription-quantitative polymerase chain reaction was conducted to determine miR-135b expression levels in the peripheral blood samples of patients with AD and age-matched normal controls. The data of the present study revealed that the expression levels of miR-135b were significantly reduced in the peripheral blood of AD patients compared with normal controls $(\mathrm{P}<0.01)$. In vitro MTT analyses identified that the overexpression of miR-135b significantly enhanced the proliferation of hippocampal cells $(\mathrm{P}<0.01)$. Furthermore, in vivo analysis using a Y-maze test indicated that injection with miR-135b mimics into the third ventricle of anesthetized SAMP8 mice significantly enhanced their learning and memory capacities $(\mathrm{P}<0.01)$. Molecular mechanism investigations identified $\beta$-site APP-cleaving enzyme 1 (BACE1) as a direct target gene of miR-135b, and the latter was identified to negatively mediate the protein expression levels of BACE1 in hippocampal cells, in addition to hippocampal tissues, of SAMP8 mice. Based on the aforementioned findings, we propose that miR-135b has a neuroprotective role via direct targeting of BACE1 and, thus, may be used for the treatment of AD.
\end{abstract}

Correspondence to: Dr Yi Zhang, Department of Neurology, The First Affiliated Hospital of Xinxiang Medical University, 88 Jiankang Road, Weihui, Henan 453100, P.R. China

E-mail: xinxiangzhangyi123@163.com

Key words: Alzheimer's disease, microRNA, neuroprotective, $\beta$-site APP-cleaving enzyme 1

\section{Introduction}

Alzheimer's disease (AD), a severe age-related neurodegenerative disorder, is characterized by the accumulation of amyloid- $\beta$ (A $\beta)$ plaques and neurofibrillary tangles, synaptic and neuronal loss, and cognitive decline $(1,2)$. An estimated 5.3 million Americans have AD; 5.1 million of these are aged 65 and over, and approximately 200,000 are $<65$ years-of-age and have younger onset AD (3). In 2013, official death certificates recorded 84,767 deaths from $\mathrm{AD}$, making $\mathrm{AD}$ the sixth leading cause of death in the United States and the fifth leading cause of death in Americans aged 65 years (3). There is currently no effective treatment capable of slowing down the progression of AD.

Among the genes associated with AD, $\beta$-site APP-cleaving enzyme 1 (BACE1) is a rate-limiting enzyme for $A \beta$ production and has been demonstrated to be significantly upregulated in patients with AD (4). Furthermore, BACE1 has been implicated as a potential target for therapies against AD (4). Therefore, understanding the mechanism underlying BACE1 upregulation in $\mathrm{AD}$ may further the development of therapeutic strategies for the treatment of AD.

MicroRNAs (miRs/miRNAs), a class non-coding RNAs 18-25 nucleotides in length, have been reported to cause mRNA degradation or inhibition of protein translation through directly binding to the 3'-untranslated region (UTR) of their target mRNAs (5). Recently, several studies have implicated certain miRs in neuronal survival, proliferation, differentiation and migration $(6,7)$. Yang et al revealed that miR-29c negatively mediates the expression of DNA methyltransferase 3, which contributes to neuronal proliferation, by regulating the expression of brain-derived neurotrophic factor (6). Furthermore, the dysfunction of certain miRs has been suggested to be involved in the development of AD (8-10). Denk et al investigated the expression profiling of 1,178 miRs in cerebrospinal fluid samples from patients with $\mathrm{AD}$ and normal controls, and discrimination analysis using a combination of miR-100, miR-103 and miR-375 was able to detect AD by positively classifying controls and AD cases with 96.4 and $95.5 \%$ accuracy, respectively (8). Furthermore, Lei et al reported that the downregulation of miR-29c was correlated with increased BACE1 expression levels in sporadic Alzheimer's disease (4). Recently, Liu et al used miR microarrays to analyze the miR expression profiles of amyloid precursor protein (APP)/presenilin 1 (PS1) 
in the hippocampi of transgenic and wild-type mice, and identified that miR-135a was significantly downregulated in the hippocampi of APP/PS1 transgenic mice compared with the wild-type control, suggesting that downregulation of miR-135a may have a role in the development of AD (11). However, the exact role of miR-135b in AD still remains largely unclear.

The primary aim of the present study was to investigate the expression levels and role of miR-135b in AD. The underlying mechanism involving BACE1 was also investigated.

\section{Materials and methods}

Collection of blood samples. The present study was approved by the ethics boards of Xinxiang Medical School (Weihui, China). Blood samples from patients from The First Affiliated Hospital of Xinxiang Medical University, (Weihui, China) with $\operatorname{AD}(\mathrm{n}=25 ; 12$ male, 13 female) aged between 65 and 81 years old and age-matched normal subjects $(n=25)$ were collected from our hospital between April 2013 and March 2014. Blood samples were stored in anticoagulation tubes at $-80^{\circ} \mathrm{C}$. Patients with diabetes, heart disease, stroke and cancer were excluded from the study. Written informed consent was obtained from all participants.

Cell culture. Primary hippocampal cells (purchased from Amspring, Changsha, China), obtained from the embryonic hippocampi of senescence-accelerated mouse resistant 1 (SAMR1) mice, were cultured in Dulbecco's modified Eagle medium (Thermo Fisher Scientific, Inc., Waltham, MA, USA) with $10 \%$ fetal bovine serum (FBS; Thermo Fisher Scientific, Inc.), and $100 \mathrm{kU} / 1$ of penicillin and streptomycin (Thermo Fisher Scientific, Inc.). Hippocampal cells were cultured in a humidified atmosphere of $95 \%$ air and $5 \% \mathrm{CO}_{2}$.

Reverse transcription-quantitative polymerase chain reaction (RT-qPCR) analysis. Total RNA was extracted from the human tissue and mouse hippocampal cells using TRIzol reagent (Thermo Fisher Scientific, Inc.). A Taqman miRNA Reverse Transcription kit (Thermo Fisher Scientific, Inc.) was used to convert RNA into cDNA. A miScript SYBR-Green PCR kit (Guangzhou RiboBio Co., Ltd. Guangzhou, China) was used to determine the miRNA expression levels, according to the manufacturer's protocol. U6 was used as an endogenous control. Expression levels of mRNA were determined using the SYBR green qPCR assay (CWBio, Beijing, China) following the manufacturer's protocol. An Applied Biosystems 7500 Thermocycler (Applied Biosystems; Thermo Fisher Scientific, Inc.) The specific primers were as follows: Forward, 5'-TCT GTCGGAGGGAGCATGAT-3' and reverse, 5'-GCAAAC GAAGGTTGGTGGT-3' for BACE1; forward, 5'-ACAACT TTGGTATCGTGGAAGG-3' and reverse, 5'-GCCATCACG CCACAGTTTC-3' for GAPDH. Expression of GAPDH was used as an endogenous control. The PCR cycling conditions were as follows: $95^{\circ} \mathrm{C}$ for $5 \mathrm{~min}$, and 40 cycles of denaturation at $95^{\circ} \mathrm{C}$ for $15 \mathrm{sec}$ and annealing/elongation step at $60^{\circ} \mathrm{C}$ for $30 \mathrm{sec}$. Data were analyzed using the $2^{-\Delta \Delta \mathrm{qt}}$ method (12).

Dual luciferase reporter assay. The seed sequences of miR-135b (5'-AAGCCAUA-3') within the BACE1 3'-UTR, or the mutant binding sequences of miR-135b within the
BACE1 3'-UTR, were cloned downstream of the luciferase gene driven by the cytomegalovirus (CMV) promoter, generating Luc-BACE1 and Luc-mutant BACE1 vectors (GeneChem, Shanghai, China), respectively. The vectors used in the luciferase reporter assay were directly purchased from Amspring. Lipofectamine 2000 (Invitrogen; Thermo Fisher Scientific, Inc., Waltham, MA, USA) was used to co-transfect (for $24 \mathrm{~h}$ at $37^{\circ} \mathrm{C}$ ) hippocampal cells with Luc-BACE1 or Luc-mutant BACE1 vectors and miR-135b mimics or scramble miR-negative control (miR-NC) mimics (GeneChem Co., Ltd.), respectively. Following a transfection period of $24 \mathrm{~h}$, luciferase activity was determined using an LD400 luminometer (Beckman Coulter Inc., Brea, CA, USA).

Western blot analysis. Tissues and cells were solubilized in cold RIPA lysis and extraction buffer (Thermo Fisher Scientific, Inc.). The concentration of protein was determined using a BCA kit (Pierce Biotechnology, Inc., Rockford, IL, USA). Proteins were separated with $10 \%$ sodium dodecyl sulfate-polyacrylamide gel electrophoresis and transferred onto a polyvinylidene difluoride membrane (PVDF; Thermo Fisher Scientific, Inc.). The PVDF membrane was incubated with phosphate-buffered saline (PBS) containing 5\% non-fat milk overnight at $4^{\circ} \mathrm{C}$, then incubated with mouse monoclonal anti-BACE1 (cat no. ab201946; 1:50; Abcam, Cambridge, MA, USA) or anti-GAPDH antibody (cat no. ab8245; 1:100; Abcam) at room temperature for $3 \mathrm{~h}$. Subsequent incubation with rabbit anti-mouse secondary antibody (cat no. ab6728; 1:10,000; Abcam) at room temperature for $1 \mathrm{~h}$ was then performed. An enhanced chemiluminescence kit (Pierce Biotechnology, Inc.) was utilized to perform chemiluminescent detection. The relative protein expression levels were analyzed with Image Pro Plus software (version 6.0; Media Cybernetics, Rockville, MD, USA), represented as the density ratio versus GAPDH.

Cell proliferation assay. An MTT assay was performed to investigate cell proliferation. For each group, 5,000 hippocampal cells per well were seeded in 96-well plates and incubated for $6,12,24$ or $48 \mathrm{~h}$ at $37^{\circ} \mathrm{C}$ and $5 \% \mathrm{CO}_{2}$. Following this, MTT $(5 \mathrm{mg} / \mathrm{ml})$ was added to each well and cells were then incubated at $37^{\circ} \mathrm{C}$ for $4 \mathrm{~h}$. The medium containing MTT was then removed and $100 \mu 1$ dimethyl sulfoxide was added. Absorbance was detected at $492 \mathrm{~nm}$ using a microplate reader (Multiskan FC Microplate Photometer; Thermo Fisher Scientific, Inc.).

Animal treatment. The experimental protocol was approved by the Animal Care and Use Committee of Xinxiang Medical School, in compliance with the National Institutes of Health Guide for the Care and Use of Laboratory Animals (13). Male senescence accelerated mouse prone 8 (SAMP8) mice $(n=18$; 8 months old) were purchased from the Animal Center of Xinxiang Medical School. Mice were housed at $22 \pm 1^{\circ} \mathrm{C}$ in a 12-h light/dark cycle. The third ventricle of anesthetized mice (0.5 ml/100g 10\% chloral hydrate; Tanghua, Changsha, China) were injected with $3 \mu \mathrm{l}$ PBS containing $0.5 \mathrm{nM}$ of miR-135b mimic or scramble miR-NC mimic (GeneChem Co., Ltd. Shanghai, China). In the control group, mice received $3 \mu \mathrm{l}$ of PBS. Each group was composed of 6 mice. At $3 \mathrm{~h}$ after injection, the mice were sacrificed using cervical dislocation under 


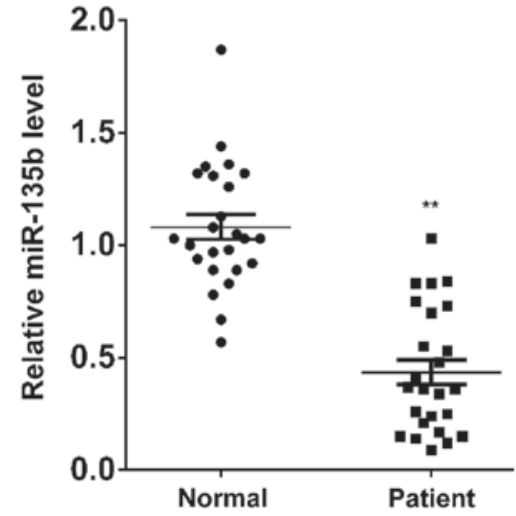

Figure 1. Reverse transcription-quantitative polymerase chain reaction was performed to determine the expression levels of miR-135b in the peripheral blood of patients with Alzheimer's disease and age-matched normal controls. ${ }^{* * *} \mathrm{P}<0.01$ vs. normal control. miR-135b, microRNA-135b.

anesthetization using $10 \%$ chloral hydrate. The heads of mice were anatomized, and the hippocampal tissues were obtained.

$Y$-maze test. The maze apparatus (DOiT, Shanghai, China) was constructed of wood painted in black with three arms. The mice were placed at the end of one arm and allowed to move freely for $10 \mathrm{~min}$. Spontaneous alternation was defined as successive entries into the three arms in overlapping triplet sets. The alternation percentage was determined as the ratio of actual alternations to maximum alternations multiplied by 100.

Statistical analysis. Data are expressed as mean \pm standard deviation. Differences between two groups were determined by Student's t-test. Statistical analyses were performed using GraphPad Prism software (version 5; Graphpad Software, Inc., La Jolla, CA, USA). $\mathrm{P}<0.05$ was considered to indicate a statistically significant difference.

\section{Results}

miR-135b levels are reduced in the blood of patients with $A D$. To reveal the role of miR-135b in AD, RT-qPCR was conducted to determine the miR-135b expression levels in the peripheral blood of patients with AD and age-matched normal controls. The data revealed that the expression levels of miR-135b were significantly reduced in the peripheral blood of AD patients compared with the normal controls $(\mathrm{P}<0.01$; Fig. 1), suggesting that the downregulation of miR-135b may have a role in the pathogenesis of AD.

miR-135b promotes the proliferation of hippocampal cells. The role of miR-135b in the regulation of hippocampal cell proliferation was subsequently investigated. Hippocampal cells were transfected with miR-135b mimics or a miR-135b inhibitor, respectively. Following transfection, RT-qPCR was performed to detect the expression levels of miR-135b in each group. As displayed in Fig. 2A, transfection with miR-135b mimics led to a significant increase in miR-135b expression levels in hippocampal cells $(\mathrm{P}<0.01)$, while transfection with the miR-135b inhibitor caused a significant downregulation
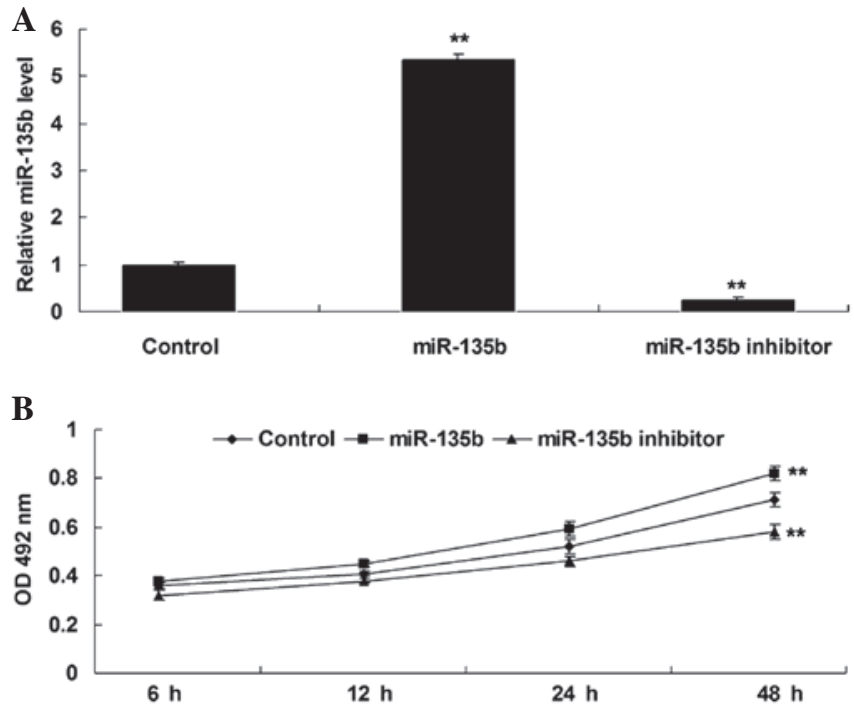

Figure 2. (A) Reverse transcription-quantitative polymerase chain reaction was performed to determine the expression levels of miR-135b in mouse hippocampal cells transfected with miR-135b mimics or an miR-135b inhibitor, respectively. (B) An MTT assay was performed to determine the proliferation of hippocampal cells in each group. Control: Non-transfected hippocampal cells. ${ }^{* *} \mathrm{P}<0.01$ vs. control. miR-135b, microRNA-135b; OD, optical density.
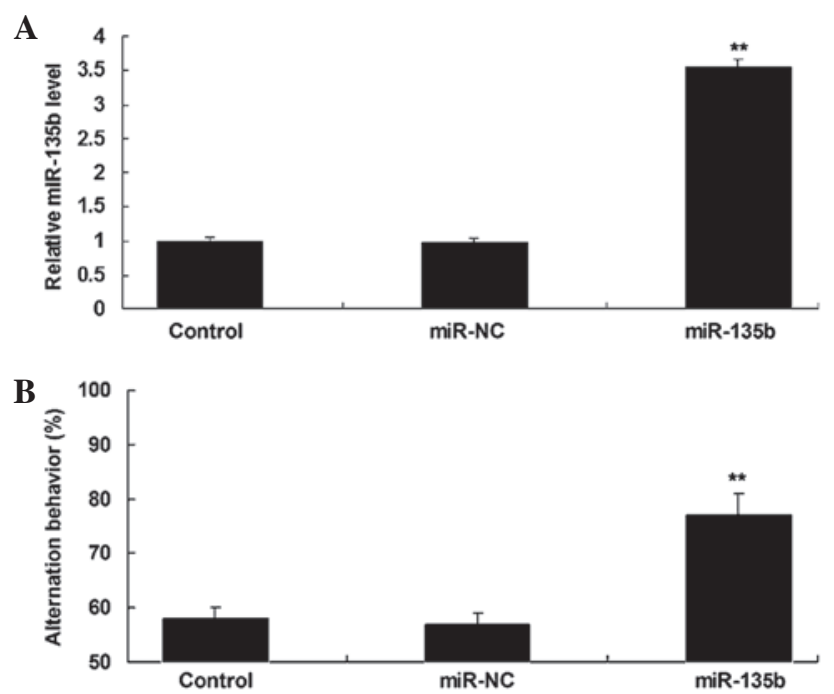

Figure 3. (A) Reverse transcription-quantitative polymerase chain reaction was performed to determine the expression levels of miR-135b in the hippocampal tissue of senescence accelerated mouse prone 8 (SAMP8) mice following injection with miR-135b mimics or scramble miR mimics (miR-NC). (B) A Y-maze test was used to measure the learning and memory behaviors of mice in each group. Control: Mice injected with equal volumes of phosphate-buffered saline. ${ }^{* *} \mathrm{P}<0.01$ vs. control. miR, microRNA; NC, negative control.

of miR-135b $(\mathrm{P}<0.01)$, compared with the control, indicating that the transfection was successful. Following this, an MTT assay was conducted to examine the proliferating capacity of hippocampal cells in each group. As demonstrated in Fig. 2B, upregulation of miR-135b significantly enhanced the proliferation of hippocampal cells, while knockdown of miR-135b significantly inhibited hippocampal cell proliferation, compared with the control group $(\mathrm{P}<0.01)$. These findings indicate that miR-135b promotes the proliferation of hippocampal cells. 


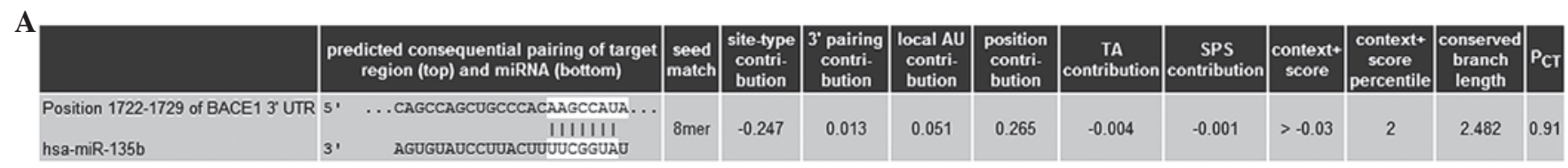

B

\author{
Wild type BACE1 3' UTR 5'...CCCACAAGCCAUA...3' \\ |||||||| \\ miR-135b 3'...UACUUUUCGGUAU...5' \\ | | | | | \\ Mutant type BACE1 3' UTR 5'...CCCACAAAUUAUA...3'
}

C

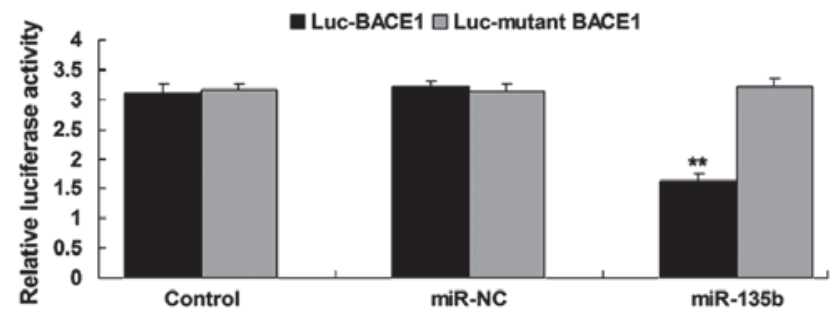

Figure 4. (A) Targetscan software predicted that BACE1 was a target gene of miR-135b. (B) The predicted miR-135b target sequence within the BACE1 3'-UTR and a mutant lacking complimentarity with miR-135b seed sequence are indicated. The seed sequences of miR-135b within the BACE1 3'-UTR, or the mutant binding sequences of miR-135b within the BACE1 3'-UTR were cloned downstream of the luciferase gene, generating Luc-BACE1 and Luc-mutant BACE1 vectors, respectively. Hippocampal cells were transfected with Luc-BACE1 or Luc-mutant BACE1 vector and miR-135b mimics or scramble miR mimics (miR-NC), respectively. (C) The luciferase activity was determined after transfection for $24 \mathrm{~h}$. Control: Hippocampal cells transfected with Luc-BACE1 or Luc-mutant BACE1 vectors alone. ${ }^{* *} \mathrm{P}<0.01$ vs. control. BACE1, $\beta$-site APP cleaving enzyme 1 ; miR, microRNA; NC, negative control; UTR, untranslated region; Luc, luciferase; $\mathrm{P}_{\mathrm{CT}}$, probability of conserved targeting.

A
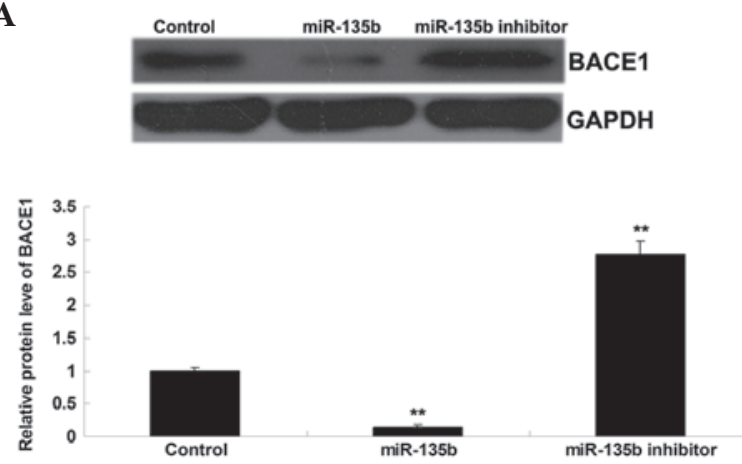

B

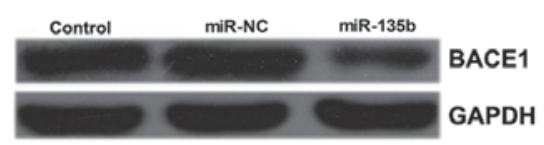

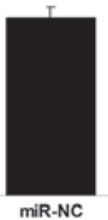

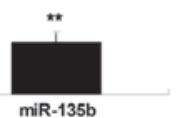

Figure 5. (A) Western blotting was performed to detect the protein expression of BACE1 in mice hippocampal cells following transfection with miR-135b mimics or miR-135b inhibitor, respectively. Control: Non-transfected hippocampal cells. ${ }^{* *} \mathrm{P}<0.01$ vs. control. (B) Western blotting was conducted to detect the protein expression of BACE1 in hippocampal tissue of mice following injection with miR-135b mimics or scramble miR mimics (miR-NC). Control: Injection with equal volume of phosphate buffered saline. ${ }^{* *} \mathrm{P}<0.01$ vs. control. BACE1, $\beta$-site APP cleaving enzyme 1; miR, microRNA; NC, negative control.

miR-135b has a neuroprotective role in SAMP8 mice. SAMP8 mice are senescence-accelerated and thus have been widely used to investigate AD $(14,15)$. In the present study, miR-135b mimics were injected into the third ventricle of SAMP8 mice to reveal the role of miR-135b in vivo. Following injection, RT-qPCR was conducted to detect the expression of miR-135b in the hippocampal tissue. As demonstrated in Fig. 3A, miR-135b was significantly upregulated in the hippocampal tissue following injection with miR-135b mimics, when compared with the control group $(\mathrm{P}<0.01)$. A Y-maze test was then performed to examine the learning and memory capacities of mice in each group. As shown in Fig. 3B, injection with miR-135b mimics into the hippocampi significantly enhanced the learning and memory behaviors of SAMP8 mice compared with the control group $(\mathrm{P}<0.01$; Fig. 3B) and indicated that miR-135b has a neuroprotective role in vivo.

BACE1 is a target gene of miR-135b. The putative targets of miR-135b that had been suggested to be associated with AD were also investigated. Bioinformatic analysis predicated that BACE1 is a putative target gene of miR-135b (Fig. 4A). To confirm this predication, the putative miR-135b target sequence within the BACE1 3'-UTR, in addition to a mutant lacking complementarity with the miR-135b seed sequence, were cloned downstream of the luciferase gene driven by the CMV promoter, generating Luc-BACE1 and Luc-mutant BACE1 vectors, respectively (Fig. 4B). Hippocampal cells were then co-transfected with Luc-BACE1 or Luc-mutant BACE1 vectors and miR-135b mimics or scramble miR mimics (miR-NC), respectively. The luciferase reporter assay data revealed that luciferase activity was only significantly decreased in hippocampal cells co-transfected with the Luc-BACE1 vector and miR-135b mimics compared with the control group $(\mathrm{P}<0.01$; Fig. 4C). However, the luciferase activity revealed no difference in other groups when compared with the control group (Fig. 4C). Therefore, the results of the present study indicate that BACE1 is a target gene of miR-135b.

BACE1 is negatively regulated by miR-135b in vitro and in vivo. As miRs negatively mediate their target genes at a post-transcriptional level, the effect of miR-135b on the protein expression levels of BACE1 in hippocampal cells of SAMR1 mice, as well as in the hippocampal tissues of SAMP8 mice, 
were investigated. Western blot analysis data revealed that the overexpression of miR-135b led to a significant decrease in the protein expression levels of BACE1, while knockdown of miR-135b caused a significant increase in the protein expression levels of BACE1 in hippocampal cells ( $\mathrm{P}<0.01$; Fig. 5A). Subsequently, the protein expression levels of BACE1 were examined in the hippocampal tissue of SAMP8 mice. As displayed in Fig. 5B, injection with miR-135b mimics caused a significant decrease in the protein expression levels of BACE1 in the hippocampal tissue of SAMP8 mice when compared with the control group $(\mathrm{P}<0.01)$. On the basis of the aforementioned data, we propose that BACE1 is negatively regulated by miR-135b in vitro and in vivo.

\section{Discussion}

It has been suggested that the dysfunction of certain miRs are involved in the development of AD. However, the exact role of miR-135b in AD, in addition to its underlying mechanism, have yet to be elucidated. The present study revealed that miR-135b was significantly downregulated in the peripheral blood of patients with AD compared with normal controls. The present study also indicated that overexpression of miR-135b significantly enhanced the proliferation of hippocampal cells, and injection with miR-135b mimics into the third ventricle of anesthetized SAMP8 mice enhanced their learning and memory capacities. Furthermore, BACE1 was identified as a target gene of miR-135b, and miR-135b negatively mediated the protein expression levels of BACE1 in hippocampal cells of SAMR1 mice, as well as in hippocampal tissues, of SAMP8 mice.

Previous studies have indicated that certain genes or miRs are significantly downregulated or upregulated in the peripheral blood or cerebrospinal fluid of patients with AD, including BACE1 (16-18). The present study revealed that the expression levels of miR-135b were significantly decreased in the peripheral blood of $\mathrm{AD}$ patients compared with those of the age-matched normal controls, suggesting that serum miR-135b levels may be used for the clinical diagnosis of AD. In agreement with the findings of the present study, Liu et al analyzed the miR hippocampi expression profiles of APP/PS1 transgenic and wild-type mice, and observed that miR-135a was significantly downregulated in the hippocampi of the transgenic mice compared with those of the wild type mice (11). Therefore, miR-135a and miR-135b may both participate in the development and progression of $\mathrm{AD}$. Hébert et al also identified that miR-29a/b-1 was significantly downregulated in the brains of patients with sporadic AD and correlated with increased BACE1 expression (19). In addition, Müller et al revealed that miR-16, miR-34c, miR-107, miR-128a and miR-146a were differentially regulated in the hippocampi of patients with AD and age-matched normal controls, while only miR-16 and miR-146a were reliably detected in the cerebrospinal fluid of patients with AD (20).

The survival and proliferation of hippocampal cells in patients with AD is significantly suppressed; thus, promoting their survival and proliferation is critical for the treatment of $\mathrm{AD}$ (21). In the present in vitro study, it was revealed that the overexpression of miR-135b significantly promotes the proliferation of hippocampal cells. Conversely, the inhibition of miR-135b expression levels significantly suppressed the proliferation of hippocampal cells, indicating that miR-135b may have a neuroprotective role. Furthermore, several other miRs have also been revealed to mediate the proliferation of neural cells. The overexpression of miR-125b, for example, inhibited the proliferation of neural stem/progenitor cells (22), and miR-34a was revealed to enhance cell proliferation and function of newly generated neurons, as well as improve behavioral outcomes (23). Furthermore, as patients with AD are characterized by learning and memory deficits (24), SAMP8 mice were used to investigate the effect of miR-135b on learning and memory behaviors in the present study. It was identified that injection with miR-135b mimics into the third ventricle significantly upregulated miR-135b expression levels in the hippocampal tissues of SAMP8 mice. This was accompanied by the upregulation of the learning and memory behaviors. Accordingly, miR-135a also appears to demonstrate a neuroprotective role in vivo.

Typically, A $\beta$ peptide is deposited in the brain of AD patients (25). BACE1 is a type I integral membrane glycoprotein and aspartic protease that is responsible for the proteolytic cleavage of APP, generating A $\beta$ peptide (25). Therefore, $\mathrm{BACE} 1$ has a critical role in AD. The expression levels of BACE1 have been revealed to be significantly increased in patients with AD (4); thus, BACE 1 is a potential therapeutic target for novel AD therapies (26). Several BACE1 inhibitors have demonstrated promising results for the treatment of $\mathrm{AD}$, which have recently been used in the human clinical trials (26). In the present study, BACE1 was identified as a direct target gene of miR-135b, and miR-135b was revealed to negatively mediate the protein expression levels of BACE1 in hippocampal cells of SAMR1 mice, as well as in the hippocampal tissues of SAMP8 mice. Therefore, miR-135b may be a potential candidate for the treatment of AD through inhibiting BACE1. In addition, several other miRs have been observed to directly target BACE1, including the miR-29 family, miR-124, miR-195 and miR-339-5p, and thus may contribute to the treatment of $\mathrm{AD}(4,27-30)$.

In conclusion, the present study indicates that miR-135b has a neuroprotective role via directly inhibiting BACE1 protein expression. Consequently, we propose that miR-135b may be utilized in the treatment of AD. Further studies are required that focus on investigating the downstream signaling pathways of miR-135b/BACE1 in the pathogenesis of AD.

\section{References}

1. Zlomuzica A, Dere D, Binder S, De Souza Silva MA, Huston JP and Dere E: Neuronal histamine and cognitive symptoms in Alzheimer's disease. Neuropharmacology: May 27, 2015 (Epub ahead of print).

2. Dezsi L, Tuka B, Martos D and Vecsei L: Alzheimer's disease, astrocytes and kynurenines. Curr Alzheimer Res 12: 462-480, 2015.

3. Alzheimer's Association: 2015 Alzheimer's disease facts and figures. Alzheimers Dement 11: 332-384, 2015

4. Lei X, Lei L, Zhang Z, Zhang Z and Cheng Y: Downregulated miR-29c correlates with increased BACE1 expression in sporadic Alzheimer's disease. Int J Clin Exp Pathol 8: 1565-1574, 2015.

5. Ambros V: The functions of animal microRNAs. Nature 431: 350-355, 2004

6. Yang G, Song Y, Zhou X, Deng Y, Liu T, Weng G, Yu D and Pan S: DNA methyltransferase 3, a target of microRNA-29c, contributes to neuronal proliferation by regulating the expression of brain-derived neurotrophic factor. Mol Med Rep 12: 1435-1442, 2015. 
7. Stary CM, Xu L, Sun X, Ouyang YB, White RE, Leong J, Li J, Xiong X and Giffard RG: MicroRNA-200c contributes to injury from transient focal cerebral ischemia by targeting Reelin. Stroke 46: 551-556, 2015.

8. Denk J, Boelmans K, Siegismund C, Lassner D, Arlt S and Jahn H: MicroRNA Profiling of CSF reveals potential biomarkers to detect Alzheimer's disease. PLoS One 10: e0126423, 2015.

9. Yang G, Song Y,Zhou X, Deng Y, Liu T, Weng G, Yu D and Pan S: MicroRNA-29c targets $\beta$-site amyloid precursor protein-cleaving enzyme 1 and has a neuroprotective role in vitro and in vivo. Mol Med Rep, 2015.

10. Zhu Y, Li C, Sun A, Wang Y and Zhou S: Quantification of microRNA-210 in the cerebrospinal fluid and serum: Implications for Alzheimer's disease. Exp Ther Med 9: 1013-1017, 2015.

11. Liu CG, Wang JL, Li L, Xue LX, Zhang YQ and Wang PC: MicroRNA-135a and -200b, potential Biomarkers for Alzheimer's disease, regulate $\beta$ secretase and amyloid precursor protein Brain Res 1583: 55-64, 2014

12. Livak KJ and Schmittgen TD: Analysis of relative gene expression data using real-time quantitative PCR and the 2(-Delta Delta C(T)) method. Methods 25: 402-408, 2001.

13. National Research Council (US) Committee for the Update of the Guide for the Care and Use of Laboratory Animals: Washington (DC). 8th edition. National Academies Press, US, 2011.

14. Hansen HH, Fabricius K, Barkholt P, Niehoff ML, Morley JE, Jelsing J, Pyke C, Knudsen LB, Farr SA and Vrang N: The GLP-1 receptor agonist liraglutide improves memory function and increases hippocampal CA1 neuronal numbers in a senescence-accelerated mouse model of Alzheimer's disease. J Alzheimers Dis 46: 877-888, 2015.

15. Takagane K, Nojima J, Mitsuhashi H, Suo S, Yanagihara D, Takaiwa $F$, Urano $Y$, Noguchi $N$ and Ishiura $S: A \beta$ induces oxidative stress in senescence-accelerated (SAMP8) mice. Biosci Biotechnol Biochem 79: 912-918, 2015.

16. Decourt B, Walker A, Gonzales A,Malek-Ahmadi M,Liesback C, Davis KJ, Belden CM, Jacobson SA and Sabbagh MN: Can platelet BACE1 levels be used as a biomarker for Alzheimer's disease? Proof-of-concept study. Platelets 24: 235-238, 2013.

17. Shaw LM, Vanderstichele H, Knapik-Czajka M,Clark CM, Aisen PS, Petersen RC, Blennow K, Soares H, Simon A, Lewczuk P et al: Alzheimer's Disease Neuroimaging Initiative: Cerebrospinal fluid biomarker signature in Alzheimer's disease neuroimaging initiative subjects. Ann Neurol 65: 403-413, 2009

18. Bibl M, Esselmann H, Lewczuk P, Trenkwalder CF, Otto M, Kornhuber J, Wiltfang J and Mollenhauer B : Combined analysis of CSF Tau, A $\beta 42, A \beta 1-42 \%$ and $A \beta 1-40 \%$ in Alzheimer's disease, dementia with Lewy bodies and Parkinson's disease dementia. Int J Alzheimers Dis 2010: Article ID 761571, 2010.
19. Hébert SS,Horré K,NicolaïL,Papadopoulou AS, Mandemakers W, Silahtaroglu AN, Kauppinen S, Delacourte A and De Strooper B: Loss of microRNA cluster miR-29a/b-1 in sporadic Alzheimer's disease correlates with increased BACE1/beta-secretase expression. Proc Natl Acad Sci USA 105: 6415-6420, 2008.

20. Müller M, Kuiperij HB, Claassen JA, Küsters B and Verbeek MM: MicroRNAs in Alzheimer's disease: Differential expression in hippocampus and cell-free cerebrospinal fluid. Neurobiol Aging 35: 152-158, 2014.

21. Moon M, Cha MY and Mook-Jung I: Impaired hippocampal neurogenesis and its enhancement with ghrelin in 5XFAD mice. J Alzheimers Dis 41: 233-241, 2014.

22. Cui Y, Xiao Z, Han J,Sun J, Ding W, Zhao Y, Chen B, Li X and Dai J: MiR-125b orchestrates cell proliferation, differentiation and migration in neural stem/progenitor cells by targeting Nestin. BMC Neurosci 13: 116, 2012

23. Mollinari C, Racaniello M, Berry A, Pieri M, de Stefano MC, Cardinale A, Zona C, Cirulli F, Garaci E and Merlo D: miR-34a regulates cell proliferation, morphology and function of newborn neurons resulting in improved behavioural outcomes. Cell Death Dis 6: e1622, 2015.

24. Buckley RF, Ellis KA, Ames D, Rowe CC, Lautenschlager NT, Maruff P, Villemagne VL, Macaulay SL, Szoeke C, Martins RN et al: Phenomenological characterization of memory complaints in preclinical and prodromal Alzheimer's disease. Neuropsychology 29: 571-581, 2015.

25. Vassar R, Kuhn PH, Haass C, Kennedy ME, Rajendran L, Wong PC and Lichtenthaler SF: Function, therapeutic potential and cell biology of BACE proteases: Current status and future prospects. J Neurochem 130: 4-28, 2014.

26. Yan R and Vassar R: Targeting the $\beta$ secretase BACE1 for Alzheimer's disease therapy. Lancet Neurol 13: 319-329, 2014.

27. Long JM, Ray B and Lahiri DK: MicroRNA-339-5p down-regulates protein expression of $\beta$-site amyloid precursor protein-cleaving enzyme 1 (BACE1) in human primary brain cultures and is reduced in brain tissue specimens of Alzheimer disease subjects. J Biol Chem 289: 5184-5198, 2014.

28. Zhu HC, Wang LM, Wang M, Song B, Tan S, Teng JF and Duan DX: MicroRNA-195 downregulates Alzheimer's disease amyloid-beta production by targeting BACE1. Brain Res Bull 88: 596-601, 2012.

29. Roshan R, Ghosh T, Gadgil M and Pillai B: Regulation of BACE1 by miR-29a/b in a cellular model of Spinocerebellar Ataxia 17. RNA Biol 9: 891-899, 2012

30. Fang M, Wang J, Zhang X, Geng Y, Hu Z, Rudd JA, Ling S, Chen $\mathrm{W}$ and Han $\mathrm{S}$ : The miR-124 regulates the expression of BACE1 $/ \beta$-secretase correlated with cell death in Alzheimer's disease. Toxicol Lett 209: 94-105, 2012. 